\title{
Percepción de profesores de la ciudad de Puebla, México, hacia la evaluación docente
}

\author{
Sandoval Castillo Nelly Patricia ${ }^{1}$, Ceja Oseguera Salvador ${ }^{2 *}$
}

${ }^{1}$ El Colegio de Puebla A.C.

${ }^{2}$ Universidad Popular Autónoma del Estado de Puebla.

\begin{abstract}
Resumen
La evaluación docente es clave para la mejora continua del proceso enseñanza-aprendizaje en todos los niveles educativos; para que sea efectiva, se requiere contar con la disposición de los evaluados, por lo que se consideró conveniente investigar cuál es la percepción de los docentes ante la evaluación de su labor profesional. Se diseñó una investigación no experimental, descriptiva y transversal simple, cuyo objetivo consistió en analizar la percepción que tienen los profesores de la evaluación de que son objeto. Se revisaron los conceptos de evaluación educativa y evaluación docente y posteriormente se construyó un cuestionario que se aplicó a 68 profesores. Los resultados obtenidos muestran una percepción negativa en docentes que tienen de 6 a 25 años de experiencia, pero positivas en quienes tienen 5 años o menos. Predomina la visión de la evaluación como medio de control y sanción, aunque más del 25\% la ve de manera más optimista. Se encontró que las mujeres, en una relación de 3 a 1 con respecto a los hombres, manifiestan estar más de acuerdo con los resultados obtenidos en su evaluación.
\end{abstract}

Palabras clave: Evaluación, Evaluación de la educación, Evaluación docente, Percepción.

\section{Perception of teachers from the city of Puebla, Mexico, towards teacher evaluation}

\begin{abstract}
Teacher evaluation is key to continuous improvement of the teaching-learning process at all educational levels; to be effective, it is necessary to have the disposition of those evaluated, so it was considered convenient to investigate what is the perception of teachers when evaluating their professional work. A non-experimental, descriptive and simple cross-sectional research was designed, the objective of which was to analyze the social representation which teachers have of the evaluation they are subject to. The concepts of educational evaluation and teacher evaluation were reviewed and later a questionnaire was constructed that was applied to 68 teachers. The results obtained show a negative perception in teachers who have 6 to 25 years of experience, but positive in those with 5 years or less. The vision of evaluation as a means of control and sanction predominates, although more than $25 \%$ see it in a more optimistic way. It was found that women, in a ratio of 3 to 1 with respect to men, state that they agree with the results obtained in their evaluation.
\end{abstract}

Keywords: Evaluation, Evaluation of education, Teacher evaluation, Perception. 


\section{INTRODUCCIÓN}

La evaluación siempre ha sido un elemento central en el proceso educativo y en la administración escolar. Se evalúa a los estudiantes, a las escuelas, al currículum, a las estrategias de enseñanza y de aprendizaje, y por supuesto que también se evalúa al docente. Aunque todo tipo de evaluación provoca cierta desazón en quienes son evaluados, esta incomodidad se vive de manera especial en quienes se dedican a la docencia. El docente, evaluador por excelencia, es de los que se muestra más receloso cuando debe ser evaluado.

Para mejorar es necesario evaluar, pues de lo contrario no habría cambio, ni innovación, que ayude a perfeccionar el desarrollo profesional docente. Pero para confrontar los diferentes procesos de evaluación es conveniente conocer con qué actitud son asumidos esos cambios por los docentes y qué valor se les reconoce (Camilloni, 2009). Dentro de la evaluación educativa, la dedicada a los docentes se presenta como una modalidad necesaria, pero al mismo tiempo no siempre bien vista. Se le llega a ver como un medio de control, como un proceso inútil o como un acto burocrático que sólo busca justificar la planeación institucional (Gallardo, 2015).

La importancia de conocer la percepción que tienen los docentes de la evaluación que se les hace, se debe a que los posibles beneficios que brinda dependen en gran medida de la actitud que asuman hacia ella. Por esta razón es que se planteó como problema central de esta investigación el siguiente: ¿Cuál es la percepción de los profesores con respecto a la evaluación a la que son sometidos?

El objetivo de la investigación fue identificar y analizar la percepción que tienen los profesores de la ciudad de Puebla hacia la evaluación que se les hace. Los sujetos investigados fueron docentes que trabajan en la zona metropolitana de la ciudad de Puebla y que laboran en diferentes niveles educativos (desde preescolar hasta profesional), tanto en el sector público como en el privado. Este grupo fue seleccionado debido a la diversidad que representan en edad, experiencia y género, con la intención de conocer los contrastes en sus 'puntos de vista hacia este tema.

En la encuesta de ingreso realizada por el Instituto Nacional para la Evaluación de la Educación (INEE) en 2018, se expone información que muestra un alto porcentaje de aceptación por parte de los profesores, entre $70 \%$ y $87 \%$, hacia los diferentes procesos de evaluación, tanto para el ingreso como para la promoción. Guzmán (2018) considera que en México la experiencia vivida de la evaluación docente está más referenciada a la forma de evaluar, lo cual poco cumple con las exigencias sociales y con el mejoramiento de la labor educativa.

La palabra evaluar proviene del francés évaluer, del francés antiguo value ("valor"), proveniente del latín vale $\square$, val $\square$ re ("ser fuerte, ser valeroso"). De aquí que a la evaluación se le haya dado la connotación de valorar, calificar, medir, analizar, retroalimentar, dependiendo de la teoría adoptada (Ogawa y Collom, 2008).

El término evaluación educativa debe su nombre a Ralph Tyler (1950) quien la define ya como proceso en el cual debe haber congruencia entre los objetivos y los logros alcanzados, según señala Alcaráz (2014). Algunas otras definiciones la señalan como «una fase de control que tiene como objeto no sólo la revisión de lo realizado sino también el análisis sobre las causas y razones para determinados resultados... y la elaboración de un nuevo plan en la medida que proporciona antecedentes para el diagnóstico» (Duque, 2013, p. 167). Por su parte, el Joint Committeeon Standardsfor Educational Evaluation señala que «la evaluación es el enjuiciamiento sistemático de la validez o mérito de un objeto» (Gullickson, 2013). Stufflebeam y Shinkfield (2000), consideran que la evaluación es un proceso complejo pero inevitable. Es una fuerza positiva que «sirve al progreso y se utiliza para identificar los puntos débiles y fuertes, y para tender hacia una mejora» (p. 20).

En México, el Consejo Nacional de Evaluación de la Política de Desarrollo Social (CONEVAL, 2014) define a la evaluación como: " [...] el análisis sistemático e imparcial de una intervención pública cuya finalidad es determinar la pertinencia y el logro de sus objetivos y metas, así como la eficiencia, eficacia, calidad, resultados, impacto y sostenibilidad" ( p. 6)

La evaluación fue definida por la Asociación Nacional de Universidades e Instituciones de Educación Superior de la República Mexicana A.C. (ANUIES), desde 1984, y por la Comisión Nacional para la Evaluación de la Educación Superior (CONAEVA), en 1989, como un proceso continuo, integral y participativo que permite identificar una problemática, analizarla y explicarla mediante información relevante. Como resultado proporciona juicios de valor que sustentan la consecuente toma de decisiones. Con la evaluación se busca el mejoramiento de lo que se evalúa y se tiende a la acción (Zorrilla, 1917).

Entre los principales teóricos de la evaluación y de la evaluación educativa, elaborados históricamente por los más destacados autores, se encuentran las siguientes: para Ralph Tyler, citado en Garduño (2019) es un proceso que determina hasta qué punto se han conseguido los objetivos educativos. Cronbach (1963) la concibe como un proceso 
de recopilación y utilización de la información para tomar decisiones. Scriven (2004) le agrega el sentido de valoración como una de sus principales cualidades. A Bloom (1971) le interesa determinar si en realidad se producen ciertos cambios en los alumnos, así como establecer el grado de cambio en cada estudiante. Para Alkin (2013) es un proceso de reconocer las áreas de decisión importantes, seleccionar la información apropiada y analizar la información apropiada para transmitir datos que ayuden a tomar decisiones.

El clásico de la evaluación educativa Stufflebeam (2003), la ve como un proceso de diseñar, obtener y proveer información útil para juzgar entre alternativas de decisión acerca de un programa educacional. Coll (1983) la ve como como un conjunto de actividades que nos llevan a emitir un juicio sobre una persona, objeto, situación o fenómeno en función de unos criterios previamente establecidos y con vistas a tomar decisiones. Gimeno (2008) señala que es un proceso por medio del cual alguna o varias características de un alumno, de un grupo de estudiantes, de un ambiente educativo, de objetivos educativos, de materiales, de profesores, programas, etcétera, reciben la atención del que evalúa, se analizan y valoran sus características y condiciones en función de unos criterios o puntos de referencia para emitir un juicio que sea relevante para la educación. Finalmente, para Mateo (2006) es una recogida de información orientado a la emisión de juicios de mérito o de valor respecto de algún sujeto, objeto o intervención con relevancia educativa. Este proceso deberá ir necesariamente asociado a otro de toma de decisiones encaminado a la mejora u optimización.

La evaluación en general se encuentra estrechamente ligada con la evaluación educativa, a tal grado de que muchas veces se considera obvio que una incluye a la otra. Para fines de esta investigación, asumimos la definición proporcionada por Gimeno (2008), quien la considera como un

proceso por medio del que alguna o varias características de un alumno, de un grupo de estudiantes, de un ambiente educativo, de objetivos educativos, de materiales, de profesores, programas, etc. reciben la atención del que evalúa, se analizan y se valoran sus características y condiciones en función de unos criterios o puntos de referencia para emitir un juicio que sea relevante para la educación (p. 45).

La evaluación docente se enfoca a quien tiene el papel de agente en el proceso de enseñanza y quien procura ser un intermediario en el aprendizaje del estudiante (Zabalza, 2015). Según Mateo (2006, p.53), esta evaluación tiene dos propósitos: uno de control del rendimiento, asociado a la obtención de buenos resultados, y otro centrado en la mejora del propio docente, pues permite la retroalimentación con el fin de mejorar profesionalmente. Otros autores (Scriven, 2004; Mateo, 2006; Alkin, 2013) incluyen la detección de necesidades, la posibilidad de la innovación educativa, la promoción laboral, el incremento salarial y la obtención de distinciones o, en su defecto, sanciones.

Señala Danielson (2007), citado en Isoré (2010, p. 10) que los docentes pueden ser evaluados de acuerdo con los siguientes contenidos: Conocimientos, destrezas, competencias, efectividad, productividad y profesionalidad.

Debido a que es un tema que despierta posturas encontradas, a continuación señalamos algunos argumentos de quienes están a favor y en contra de la evaluación docente, de acuerdo con lo señalado por Isoré (2010). Los que se expresan a favor, señalan los siguientes argumentos: a) El sistema actual debe hacer responsable a los docentes por su práctica y su desempeño; b) Las autoridades y los padres tienen el derecho a que sus hijos reciban educación de calidad; c) Las evaluaciones permiten la identificación de quienes tienen un buen desempeño, de manera similar a las empresas; d) Son esenciales para hacer más atractiva la profesión; e) Es necesario tomar medidas ante los malos docentes; f) Las evaluaciones permiten que los docentes identifiquen sus fortalezas y deficiencias y evalúen sus necesidades de desarrollo profesional; g) Se requiere eficientar el tiempo, la energía y el dinero; $\mathrm{h}$ ) Las evaluaciones mejoran la práctica de los docentes y el aprendizaje de los estudiantes; i) Las evaluaciones del desempeño docente mejoran la cooperación entre los docentes.

Los que están en contra de la evaluación docente, exponen los siguientes argumentos: a) No es posible determinar el desempeño docente en forma objetiva y la "buena enseñanza" puede manifestarse de diversas formas; b) La enseñanza requiere un ambiente alejado de presiones políticas, sociales y financieras; c) Los mecanismos empresariales no tienen lugar en la educación; d) Los docentes no están motivados por recompensas financieras, sino por aspectos "intrínsecos" (el deseo de enseñar); e) Algunos docentes pueden desmotivarse como consecuencia de los procedimientos de evaluación; f) Se estigmatiza a los docentes; g) Los sistemas de evaluación son caros y emplean demasiado tiempo; h) Las evaluaciones producen una serie de efectos negativos tales como la restricción del currículo y el descuido a los estudiantes; i) Las evaluaciones del desempeño docente reducen la cooperación entre los docentes debido a los efectos de competencia.

\section{MATERIALES Y MÉTODOS}

Se diseñó una investigación no experimental, descriptiva, transversal simple, para analizar la percepción que tienen los profesores de la ciudad de Puebla, México, hacia la evaluación de su desempeño profesional. 
Se inició con una revisión documental de los conceptos de evaluación y evaluación docente. Posteriormente se construyó y aplicó un cuestionario a docentes, el cual consta de 16 ítems, algunos con respuesta dicotómica y otros en una escala Likert 5, en donde la posición 1 corresponde a Totalmente en desacuerdo, mientras que la posición 5 significaba Totalmente de acuerdo.

Se aplicó una prueba piloto a 34 profesores que estudian un posgrado en educación. El grado de confiabilidad obtenido, con base en el Alfa de Cronbach, fue de .902, por lo que el instrumento resulta confiable. El cuestionario fue aplicado a 68 docentes, quienes cuentan con las siguientes características:

Todos han sido evaluados al menos una vez en los últimos cinco años; el 79\% por instituciones públicas (SEP, INEE, su institución) y el $21 \%$ por instituciones privadas.

- El 76.5\% fueron hombres y el 23.5\% mujeres.

- El $17.6 \%$ eran menores de 30 años, el $70.5 \%$ tenía de 31 a 59 y el 3\% más de 60 años.

- El $78 \%$ contaba sólo con licenciatura y el $22 \%$ con posgrado.

La mayoría de los encuestado (72.1\%) son considerados docentes experimentados, pues cuentan de 6 a 24 años laborando en la educación. Quienes tienen 5 años o menos son considerados de poca experiencia (14\%) y quienes tienen 25 años o más son considerados con amplia experiencia.

\section{Supuestos}

Con base en la bibliografía revisada, se plantearon los siguientes supuestos:

- Los profesores perciben de manera favorable la evaluación docente.

- Los profesores perciben la evaluación docente como una oportunidad de mejorar en su desempeño profesional.
- Los profesores no perciben la evaluación docente como un instrumento para controlarlos.

\section{RESULTADOS Y DISCUSIÓN}

De acuerdo con el sexo. Al cuestionárseles si estaban de acuerdo con el resultado que habían obtenido cuando fueron evaluados, el $68.8 \%$ de los hombres y el $52 \%$ de mujeres manifestó estar en desacuerdo. Sólo el $6.3 \%$ de hombres y el $21.2 \%$ de mujeres se mostró de acuerdo; es notorio que la aprobación por parte de las mujeres supera a la de los hombres en una relación de 3 a 1, lo cual abre la pregunta: ¿A qué se debe esta marcada diferencia?

Con relación a la edad. En este rubro se notó una diferencia significativa entre los más jóvenes (de 20 a 30 años), quienes en su totalidad manifestaron estar de acuerdo, contra los mayores de 31 y en especial mayores de 41, quienes suman un desacuerdo del $60.4 \%$ y un $88 \%$ respectivamente.

Con relación al nivel de estudios. En este sentido, el acuerdo o desacuerdo no es muy notorio; entre quienes poseen el grado de licenciatura hay una tendencia más hacia el desacuerdo, pero entre los de posgrado está dividido el acuerdo y desacuerdo con un 50\% cada uno.

Con relación a la experiencia docente. En este punto se encontró un dato curioso: se manifiestan de acuerdo los de menor experiencia $(77.8 \%)$ y los de mayor experiencia (66.6\%), pero no así quienes tienen entre 6 y 26 años, quienes están en desacuerdo: los de 6 a 15 años de experiencia con un $62.9 \%$ y los de 16 a 25 años con un desacuerdo del $77.8 \%$ (Tabla 1).

Se les preguntó a los profesores si consideraban adecuados los criterios con los cuales fueron evaluados, y un $64.7 \%$ manifestó su rechazo, contra un $17.7 \%$ que los aceptó; el resto $(17.6 \%)$ no se manifestó ni a favor ni en contra. De igual manera el $75 \%$ manifestó haber tenido dificultades cuando fueron evaluados.

Tabla 1. Satisfacción con el resultado de la evaluación con base a la experiencia docente.

\begin{tabular}{|lcccc|}
\hline & 1 Totalmente Desacuerdo & 2 Desacuerdo & 3 De Acuerdo & 4 Totalmente Acuerdo \\
\hline Menos de 5 & $8.30 \%$ & $0.00 \%$ & $37.80 \%$ & $40.00 \%$ \\
\hline $6-15$ años & $29.60 \%$ & $33.30 \%$ & $29.60 \%$ & $7.40 \%$ \\
\hline $16-25$ años & $37.80 \%$ & $40.00 \%$ & $13.60 \%$ & $9.10 \%$ \\
\hline $26-35$ años & $16.70 \%$ & $16.70 \%$ & $33.30 \%$ & $33.30 \%$ \\
\hline
\end{tabular}

En esta tabla se puede observar que quienes más desacuerdo manifiestan con los resultados de la evaluación son quienes tienen laborando entre 6 y 25 años y que quienes manifiestan si la aprueban son quienes tienen menos de 5 años o más de 26 años. 
Se les preguntó si percibían la evaluación docente como un proceso, es decir, como algo dinámico, una acción continua que responde a ciertas finalidades, o como un suceso, es decir, algo que ocurre sólo en un momento dado. Para la mayoría (76.5\%) la evaluación es un proceso

Se les preguntó a los profesores si percibían la evaluación como una oportunidad de detectar su situación profesional en ese momento o sólo como una obligación burocrática o como una oportunidad de retroalimentación. La mayoría (el 55.8\%) no la veían como una oportunidad para detectar oportunidades; el $72 \%$ la veía como un obligación con fines burocráticos que es indispensable cumplir; sin embargo, y aunque no fueron mayoría, el $39.7 \%$ la veía como una oportunidad de retroalimentación, contra el $51.4 \%$ que no le reconocía este tipo de aportación.

En una pregunta similar a la anterior, cuando se les preguntó si con la evaluación se lograba mejorar la educación, el $57.4 \%$ señaló que no. Tampoco creen que los profesores mejor evaluados favorezcan más el aprendizaje de sus alumnos (77.9\% dice que no, contra $22.1 \%$ que dice que sí).

Algo destacado fue su descontento ante la obligatoriedad de la evaluación docente, pues el $60.3 \%$ se manifestó en contra de esto (Tabla 2).

Tabla 2. Postura ante la obligatoriedad de la evaluación docente

\begin{tabular}{lcc} 
& Frecuencia & Porcentaje \\
\hline Totalmente desacuerdo & 13 & 19.1 \\
\hline Desacuerdo & 28 & 41.2 \\
\hline Indiferente & 1 & 1.5 \\
\hline De acuerdo & 16 & 23.5 \\
\hline Totalmente de acuerdo & 10 & 14.7 \\
\hline Total & 68 & 100
\end{tabular}

En esta tabla se puede observar que la mayoría (60.3\%) rechaza la obligatoriedad de la evaluación docentes y que sólo el 38.2 está de acuerdo con ella.

Al revisar su postura ante las consecuencias de la evaluación, la gran mayoría la percibe como un medio de control (79.5\%) y como un instrumento que puede acarrearles sanciones (57.3\%). No la ven como una actividad que favorezca su desarrollo profesional $(51.5 \%)$ ni como un proceso con consecuencias formativas (52.9\%). Es decir, que predomina una percepción negativa (Tabla 3).

\section{Supuestos:}

a) El supuesto de que «Los profesores perciben de manera favorable la evaluación docente» se rechaza, debido a que predomina más el temor ante las posibles sanciones y al control que se puede ejercer con esta actividad. Pero resulta interesante señalar que la visión es más positiva en los profesores jóvenes y en quienes tienen menos experiencia, lo cual puede deberse a que varios de ellos accedieron a laborar en la docencia gracias a los exámenes de selección que se aplicaron en ese entonces, a diferencia de quienes poseen mayor edad o llevan más años trabajando, quienes pudieron haber accedido a una plaza por otros medios.

b) El supuesto de que «Los profesores perciben la evaluación docente como una oportunidad de mejorar en su desempeño profesional» se rechaza, pues no creen que mejore su desempeño laboral gracias a este proceso. Incluso no están de acuerdo en que los profesores mejor evaluados son quienes tienen mejores resultados.

Tabla 3. Consecuencias de la evaluación

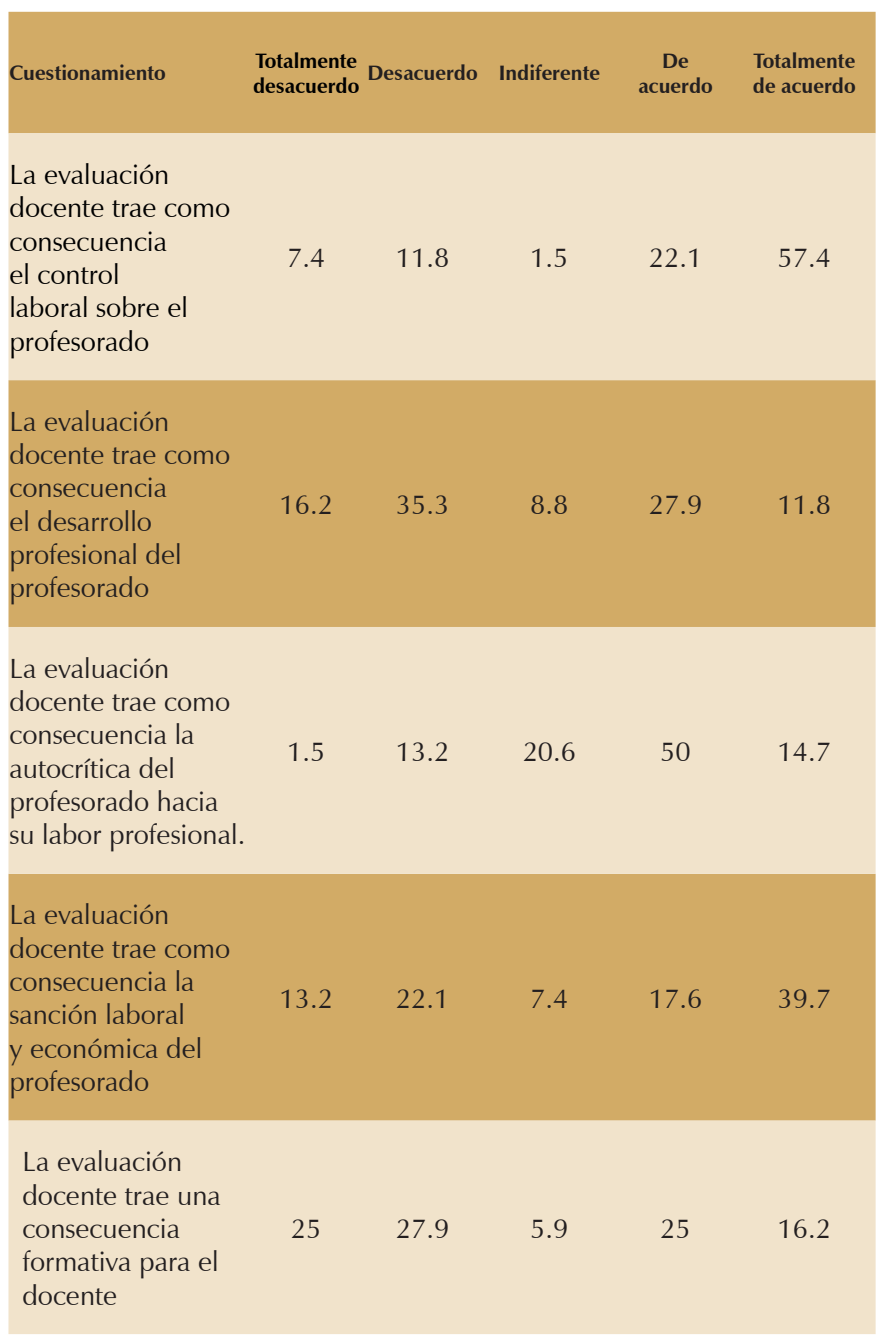

Aquí se puede observar una visión negativa hacia la evaluación docente, pues el $79.5 \%$ la percibe como un medio de control, el $57.3 \%$ como instrumento sancionador y más del $50 \%$ no le ve beneficios formativos. 
c) El supuesto de que «Los profesores no perciben la evaluación docente como un instrumento para controlarlos» no se acepta, debido a que la mayoría expresa directa e indirectamente temor de ser sancionados si obtienen malos resultados.

\section{CONCLUSIONES}

Es necesario revalorar la importancia de la evaluación y verla no como un suceso aislado, sino como un proceso que abarca desde el inicio hasta el final del acto educativo. Conviene que se reconozca que su objetivo es la promoción del aprendizaje, por lo que requiere la participación activa y positiva de los docentes.

Se encontró que los docentes evaluados oponen una resistencia muy significativa al momento de pasar por este proceso, ya que lo ven como un mecanismo de control, que no les trae como consecuencia necesariamente el ascenso escalafonario en el magisterio, una mejora salarial o el reconocimiento social por la labor que se lleva a cabo.

Y si bien la mayoría concibe la evaluación docente como un instrumento de control, que no brinda reconocimiento social ni ayuda al mejoramiento profesional, es de destacar que existe un porcentaje considerable, mayor al 25\%, cuya percepción tiende a ser más favorable al proceso de evaluación. Dentro de los encuestados, quienes se muestran de acuerdo con el proceso de evaluación son los docentes que trabajan en el nivel básico.

Es fundamental crear las condiciones laborales para que la evaluación del desempeño docente se convierta en una herramienta indispensable para gestionar la calidad, así como implementar modelos de evaluación actualizados, con el fin de que se enriquezca el desarrollo humano y profesional del docente, con la intención de formar bien a sus estudiantes.

\section{REFERENCIAS}

Alcaráz, N. 2014. Un viejo trío de conceptos: Aprendizaje, currículum y evaluación. Revista Aula de Encuentro. Vol. 16 No.: pp.67-68

Alkin, M. 2012. Evaluation roots. A Wider Perspective of Theorists' Views and Influences, Second Edition. Edit. SAGE. País USA

Camilloni, A. 2009. Estándares, evaluación y currículo. Archivos de Ciencias de la Educación (4⿳亠口了 época). Año 3, no. 3, pp. 55-68. País. Argentina

Coll, C. 2004. Psicología y currículum: una aproximación psicopedagógica a la elaboración del currículum escolar. Paidos Ibérica. Madrid.
Conde, C. 2017. Avances y retrocesos de la evaluación en México. La perspectiva de los evaluadores. Revista Política y cultura. 47: 9-40.

Consejo Nacional de Evaluación de la Política de Desarrollo Social. 2014. La política de evaluación en México: 10 años del Coneval. Coneval. México.

Cronbach, L 1963. Coefficient alpha and the internal structure of tests. Psychometrika, 16-3: 297-334.

Danielson, C. 2017. New Trends in Teacher Evaluation. Educational Leadership. 58-8:12-15

Díaz-Barriga, A. 1998. .Evaluación Académica: Organismos Internacionales y Política Educativa. UNAM. México.

Díaz-Barriga, A. 2004. La evaluación de la docencia. Su generación, su adjetivación y sus retos. Capítulo II en Rueda, M. (coord.). ¿Es posible evaluar la docencia en la universidad? Experiencias en México, Canadá, Francia, España y Brasil., ANUIES, pp 63-76, México.

Díaz-Barriga, F., Hernández G. 2018. Estrategias docentes para un aprendizaje significativo. McGraw Hill. México.

Duque, R. 1993. La evaluación en la ES Venezolana. Planiuc. X-17-18.

Gallardo, K., Ramos, A., Camacho, D., Gil, M. 2015. Evaluación de los estudiantes: las prácticas institucionales y docentes vistas desde el marco de estándares internacionales. Apertura, Vol. 7, Núm. 2

Garduño. M 2019. El modelo Tyleriano de curriculum y el papel de los objetivos conductuales. ¿Tyler fue realmente conductista? Revista Historia de la Educación. Vol. $22 \mathrm{~N}^{\circ}$ 22, enero -junio.

Gimeno Sacristán, J. (2008). Comprender y transformar la enseñanza. Morata. Madrid.

González, L. y Ayarza, H., 2016. Calidad, evaluación institucional y acreditación en la educación superior en la región Latinoamericana y del Caribe. Documento central. La educación superior en el siglo XXI. Visión de América Latina y el Caribe. Conferencia Regional Políticas y Estrategias para la Transformación de la Educación Superior en América Latina y el Caribe, La Habana, Cuba, 1996. Caracas: CRESALC-UNESCO.

Gullickson, A. 2013 The student Evaluation Standards. Join Committee on Standards for Educational Evaluation. Thousand Oaks, CA: Corwin Pres. ETS Educational Policy Leadership Institute

Guzmán-Marín, F. 2018. La Experiencia de la Evaluación Docente en México: Análisis Crítico de la Imposición del Servicio Profesional Docente. Revista Iberoamericana de Evaluación Educativa, 2018, 11(1), 135-158.

INEE. 2018. Encuestas de Opinión Docente. Ingreso y Promoción. Ciudad de México. Abril de 2019. https:// cutt.ly/arTKJm9.

Isoré, M. 2010. Evaluación docente: prácticas vigentes en los países de la OCDE y una revisión de la literatura. París: PREAL: Documentos № 46.

Mateo, J. 2006. La evaluación educativa, su práctica y otras metáforas. Alfaomega. Barcelona. 
Ogawa, R. y Collom, E. 2008. Educational indicators: What are they? How can schools and schools districts use them? California Educational Research Cooperative. Riverside

Scriven 2004. Evaluation as a discipline. Studies in Educational Evaluation. 20, 147-166

Schutz, A., Thomas, L. 2001. Las estructuras del mundo de la vida. Amorrortu. Buenos Aires.

Stufflebeam. 2003. Métodos cuantitativos y cualitativos de la investigación evaluativa. Morata. Madrid.
Stufflebeam, D., Shinkfield, A. 2000. Evaluación sistemática - Guía teórica y práctica. Paidós. España.

Zabalza, M. \& Zabalza, M. 2012. Profesores y profesión docente. Entre el "ser" y el "estar". Narcea. Madrid.

Zorrilla, M. 2017. Consejo Social Consultivo de Evaluación de la Educación. 2a . Sesión Ordinaria Servicio Profesional Docente. Ciudad de México. https://cutt.ly/orTKTGN. 